\title{
Ioannis N. Mavridis: Stereotactic brain microanatomy: mathematical principles and applications (Neuroanatomy Research at the Leading Edge)
}

\author{
Nova Biomedical - Nova Science Publishers, New York, 2017, xxxvi + 128 p, 33 \\ illustrations, format $15.6 \times 23 \mathrm{~cm}$, ISBN: 978-1-53612-366-1, ISBN: 978-1-53612-380-7 \\ (e-Book)
}

\section{Efstratios-Stylianos Pyrgelis ${ }^{1}$ (D)}

Received: 24 October 2017 / Accepted: 26 October 2017 / Published online: 3 November 2017

(c) Springer-Verlag France SAS 2017

Author of several articles on human stereotactic neuroanatomy, Dr. Ioannis N. Mavridis, aimed to present the mathematical principles and applications of stereotactic brain microanatomy, a new field in human anatomy, in a concise and comprehensive manner.

This book actually explains the robust mathematical principles of stereotactic brain microanatomy and its main clinically significant applications to modern stereotactic and functional neurosurgery. Stereotactic space of the human brain is defined according to the principles of analytic geometry, enabling the application of mathematical formulas to define brain areas. Compliance to these principles can offer a safe and mathematically accurate way to target even the smallest structures of the brain. Its clinical applications to neurosurgery are expected to be numerous and useful particularly for minimally invasive procedures.

This work, that presents with simplicity a difficult subject, is structured in seven subsequent chapters which are organized as follows: Origin of Stereotactic Space; Axes of Stereotactic space; Planes in Stereotactic Space; Points in Stereotactic Space; Other Coordinate Systems; The Concept of Stereotactically Standard Areas; The Example of Mavridis' Area.
Dr. Ioannis N. Mavridis is already known in the fields of neuroanatomy, stereotactic anatomy, and neurosurgical anatomy from his detailed studies, primarily focused on the human nucleus accumbens, including the discovery of its atrophy in Parkinson's disease.

Applications of fundamental scientific principles are of paramount importance in modern medicine, which is characterized by rapid changes, new technological developments, and emerging challenges. In this direction, applied mathematics, where stereotactic brain microanatomy is based on, may guide stereotactic neurosurgical interventions in a highly accurate and reproducible way, providing, at the same time, a solid mathematical basis for stereotactic neurosurgery to evolve into stereotactic microneurosurgery.

Beside mathematical formulas, this nicely illustrated book contains 33 figures of unique quality, including macroscopic neuroanatomical views and elaborated mathematical figures. Clearly presented, appealing, and easy to read, this book is a useful tool for all neurosurgeons, neuroanatomists, and other scientists interested in brain anatomy and stereotaxy.
Efstratios-Stylianos Pyrgelis

stratospyrg@yahoo.gr

1 1st Department of Neurology, University of Athens School of Medicine, 'Eginition' Hospital, Athens, Greece 